\title{
PERFIL EPIDEMIOLÓGICO DE MULHERES TRABALHADORAS: ANÁLISE DOS REGISTROS NO OESTE DE SANTA CATARINA
}

\section{EPIDEMIOLOGICAL PROFILE OF WORKER WOMEN: ANALYSIS OF THE RECORDS IN THE WEST OF SANTA CATARINA}

\section{PERFIL EPIDEMIOLOGICO DE ANÁLISES DE MUJERES TRABAJADORAS: ANALISIS DE LOS REGISTROS EM EL OESTE DE SANTA CATARINA}

\author{
Michelly Carla Santin ${ }^{1}$, Adriana Remião Luzardo², Milena Schneiders ${ }^{3}$, Alexandre Inácio Ramos ${ }^{4}$, \\ Kassiano Carlos Sinski ${ }^{5}$, Vander Monteiro da Conceição ${ }^{6}$
}

\section{RESUMO}

Objetivo: Analisar o perfil epidemiológico de mulheres trabalhadoras envolvidas em acidentes e/ou doenças relacionadas ao trabalho no Oeste de Santa Catarina. Método: Estudo epidemiológico transversal descritivo de caráter observacional, com coleta de dados retrospectiva. Os dados foram coletados no período de 2012 a 2016. Resultados: Os resultados mostraram que a faixa etária predominante de mulheres acidentadas são dos 20 aos 39 anos; os tipos de lesão mais prevalentes são a lesão cortante e traumatismo; quanto aos acidentes destacam-se os com material biológico, LER/DORT, intoxicações exógenas e os acidentes de trabalho não especificados. As profissões mais notificadas são: alimentador da linha de produção, faxineiro e técnico de enfermagem. Conclusão: A notificação dos agravos à saúde do trabalhador é necessária pensando no planejamento e replanejamento das ações, programas e políticas voltadas para a saúde da mulher trabalhadora que auxiliem na prevenção de acidentes e agravos e sobretudo na redução de desfechos desfavoráveis.

Descritores: Mulheres Trabalhadoras; Acidentes de Trabalho; Doenças Profissionais.

\begin{abstract}
Objective: To analyze the epidemiological profile of worker women involved in work - related accidents and / or diseases in the West of Santa Catarina. Methods: This is a descriptive crosssectional epidemiological study of an observational nature, with retrospective data collection. Data were collected between 2012 and 2016. Results: The results showed that the predominant age group of injured women are between 20 and 39 years of age; the most prevalent types of injury are cutting and trauma; As for accidents, the most notable are those with biological material, RSI/WMSD, exogenous intoxications and unspecified work-related accidents, and the most frequently reported professions include the production line feeder, the occupation of Cleaner and the Technician of nursing. Conclusion: Notification of injuries to workers' health is necessary, and there is a need to plan and re-plan actions, programs and policies aimed at the health of working women that help in the prevention of accidents and injuries and especially in reducing unfavorable outcomes.

Descriptors: Women Working; Accidents Occupational; Occupational Diseases.

\footnotetext{
${ }^{1}$ Enfermeira, Universidade Federal da Fronteira Sul, Campus Chapecó - SC.

${ }^{2}$ Enfermeira, Doutora em Enfermagem, Universidade Federal da Fronteira Sul, Campus Chapecó - SC.

${ }^{3}$ Acadêmica de Enfermagem, Universidade Federal da Fronteira Sul, Campus Chapecó - SC.

${ }^{4}$ Acadêmico de Enfermagem, Universidade Federal da Fronteira Sul, Campus Chapecó - SC.

${ }^{5}$ Acadêmico de Enfermagem, Universidade Federal da Fronteira Sul, Campus Chapecó - SC.

${ }^{6}$ Enfermeiro, Doutor em Ciências, Universidade Federal da Fronteira Sul, Campus Chapecó - SC.
} 


\section{RESUMEN}

Objetivo: analizar el perfil epidemiológico de mujeres trabajadoras involucradas en accidentes y/o enfermedades relacionadas al trabajo en el Oeste de Santa Catarina, Brasil. Método: se trata de una investigación epidemiológica transversal descriptiva de carácter observacional, con colecta de datos retrospectiva. Los datos fueron colectados en el período de 2012 a 2016 y obtuvieron una totalidad de 3.084 notificaciones relativas a los accidentes del trabajo y enfermedades relacionadas al trabajo de la mujer. Resultados: los resultados mostraron que las edades predominantes de mujeres accidentadas son de los 20 a los 39 años, los tipos de lesiones más comunes son la lesión cortante y el traumatismo; cuanto a los accidentes se destacan con material biológico, las $L E R / D O R T$, las intoxicaciones exógenas y los accidentes de trabajo no especificado y cuanto las profesiones más notificadas se evidencia el alimentador de la línea de producción, la ocupación de limpiador y la del técnico en enfermería. Conclusión: Por lo tanto, la notificación de los agravios a la salud del trabajador es necesaria, ocurriendo la necesidad en planear y replantear acciones, programas y políticas enfocadas a la salud de la mujer trabajadora que ayuden en la prevención de accidentes y agravios y sobre todo de desenlaces desfavorables. Descriptores: Mujeres Trabajadoras; Accidentes de Trabajo; Enfermedades Profesionales.

\section{INTRODUÇÃO}

O trabalho, no modelo capitalista, é determinado pelo processo de produção, no qual acidentar-se e adoecer são problemas econômicos e sociais. ${ }^{1}$ A Organização Internacional do Trabalho estimou que 2,34 milhões de pessoas morrem todos os anos em virtude de acidentes e doenças relacionados com o trabalho, sendo 2,02 milhões $(86,3 \%)$ causados por doenças profissionais e 321 mil em consequência de acidentes de trabalho. São 6.300 mortes diárias relacionadas ao trabalho, 5.500

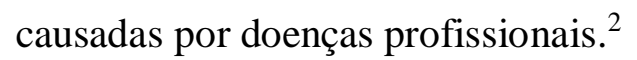

O conceito de acidente do trabalho é definido pela Lei 8.213/1991 em seu artigo 19 que estabelece: "Acidente do trabalho é o que ocorre pelo exercício do trabalho a serviço da empresa, ou ainda pelo exercício do trabalho dos segurados especiais, provocando lesão corporal ou perturbação funcional que cause morte, a perda ou redução da capacidade para o trabalho permanente ou temporário". Conforme esta mesma Lei, no artigo 20, incisos I e II... "Doenças ocupacionais são as moléstias de evolução lenta e progressiva, originárias de causa igualmente gradativa e durável, vinculadas às condições de trabalho". 3

Os acidentes e doenças relacionadas ao trabalho são agravos previsíveis e evitáveis. Contudo, tais eventos continuam acontecendo e repercutindo com forte impacto sobre a produtividade, a economia, a sociedade e a vida dos trabalhadores. Os acidentes e as mortes no trabalho estão entre as maiores problemáticas relacionadas à saúde do trabalhador no Brasil. Diante disso, o Ministério da Saúde tem criado estratégias como a implantação da Política 
Nacional de Notificação de Acidentes e Doenças no Trabalho que tem resultado na criação da Rede Nacional de Atenção à Saúde do Trabalhador (RENAST) e do Centro de Referência em Saúde do Trabalhador (CEREST). ${ }^{4}$

A inserção das mulheres no mercado de trabalho efetivou-se a partir do avanço do capitalismo industrial. Ao inserir-se no mercado de trabalho, a mulher passou a desempenhar múltiplos papéis, visto que seu papel de esposa, mãe e dona de casa é mantido até hoje, sendo que sua dedicação não é exclusiva ao lar. A mulher contemporânea, ao mesmo tempo em que é dona de casa, é também estudante e trabalhadora e além de sua realização profissional, o trabalho está ligado também à própria subsistência e de sua família, pois suas atribuições também passam a ser de ordem financeira, já que sua renda é incorporada à renda familiar. ${ }^{5}$

Um estudo realizado pelo Ministério da Previdência Social aponta que as mulheres são mais vulneráveis a doenças causadas pelo trabalho. Entre 2004 e 2013, enquanto os vínculos empregatícios tiveram um crescimento de $79 \%$ entre as mulheres, à concessão de auxílio-doença acidentário cresceu $172 \%$ entre as trabalhadoras. Entre os homens, o emprego assalariado cresceu $53 \%$ - durante o mesmo período - enquanto a concessão do auxílio-doença acidentário cresceu pouco mais de $60 \%$. Enquanto os homens apresentam maior vulnerabilidade para causas traumáticas, as mulheres afastam-se mais em decorrência de doenças relacionadas às condições ergonômicas do trabalho. $^{6}$

Frente ao exposto anteriormente questiona-se: qual o perfil epidemiológico de mulheres trabalhadoras envolvidas em acidentes e/ou doenças relacionadas ao trabalho no Oeste de Santa Catarina, Brasil?

Para responder à questão de pesquisa, formulou-se o objetivo de: Analisar o perfil epidemiológico de mulheres trabalhadoras envolvidas em acidentes e/ou doenças relacionadas ao trabalho no Oeste de Santa Catarina, Brasil.

\section{MÉTODOS}

Trata-se de um estudo epidemiológico transversal descritivo, com coleta de dados retrospectiva, de natureza explicativa e abordagem quantitativa. Realizou-se a coleta dos dados por meio da consulta aos registros de notificações do CEREST da Macrorregião do Grande Oeste, onde se analisou o perfil epidemiológico das mulheres trabalhadoras envolvidas em acidentes e/ou doenças relacionadas ao trabalho, no período de 2012 a 2016, período este disponibilizado pela instituição local do estudo considerando que o tempo de atualização 
dos sistemas de informação ocorrerem em atraso ao ano vigente.

O perfil de avaliação epidemiológica do estudo descritivo descreve a caracterização de aspectos semiológicos, etiológicos, fisiopatológicos e epidemiológicos de uma doença. São utilizados para conhecer uma nova ou rara doença, ou agravo à saúde. A direcionalidade temporal do estudo retrospectivo realiza-se a partir de registros do passado, e é seguido adiante a partir daquele momento até o presente. ${ }^{7}$

Segundo dados do Instituto Brasileiro de Geografia e Estatística, Santa Catarina possui 6.248.436 milhões de habitantes, com a estimativa de 6.910 .553 para 2016 . Nesse contexto, está o oeste catarinense como uma região conhecida pelo seu dinamismo e crescimento econômico, onde se destaca o município de Chapecó, sendo considerado a capital do oeste, com intensa atividade de indústrias responsáveis pela oferta de empregos na região. ${ }^{8}$

Para estudar a realidade da mulher trabalhadora frente aos acidentes e doenças ocupacionais, buscou-se então desenvolver este estudo no CEREST da Macrorregião do Grande Oeste, com sede em Chapecó, o qual está em funcionamento desde abril de 2007, abrangendo 76 municípios, mais de 730 mil habitantes e 15 mil $\mathrm{km}^{2}$. O referido serviço é responsável por organizar e sistematizar os dados no Sistema de Informação de Agravos de Notificação de agravos à saúde do trabalhador. A partir destas notificações o CEREST faz vigilância em saúde, vistoriando empresas, realizando ações educativas, entre outras atribuições e tem como objetivo fornecer subsídios técnicos para o SUS. Para tanto, recebe recursos do Ministério da Saúde.

É através das unidades de Saúde e do hospital que se notifica ao CEREST, por uma ficha de notificações incluída num sistema padrão. De posse das informações recebidas o fiscal sanitário do município faz uma visita à empresa. A notificação é necessária conforme Portaria do Ministério da Saúde.

Com base nestas ações desempenhadas pelo CEREST, foram acessados os registros de mulheres trabalhadoras, no período de 2012 a 2016, residentes do município de Chapecó. Os dados foram coletados de agosto a setembro de 2017, por meio de um instrumento elaborado com base nas fichas de notificação de acidentes de trabalho e nas fichas de notificação de doenças relacionadas ao trabalho, as quais notificam enfermidades como câncer, Lesão por esforço repetitivo (LER) e Distúrbio osteomuscular relacionado ao trabalho (DORT), além de transtornos mentais. 
A partir disso, foram elencadas as variáveis: idade, estado civil ou conjugal, escolaridade, número de filhos, renda, ocupação, local de trabalho, acidentes de trabalho (tipo de acidente, agente causador, sequelas) e doenças relacionadas ao trabalho. Foram considerados somente os dados do município de Chapecó/SC.

Os dados obtidos da análise dos registros foram armazenados em uma planilha eletrônica desenvolvida no programa Br Office Calc (software livre), sendo transferidos para o programa de computação Statistical Package for the Social Sciences (SPSS), versão 22.0, para análise.
Os dados foram apresentados por meio de frequência absoluta e relativa, medidas de tendência central. A pesquisa foi aprovada pelo Comitê de Ética em Pesquisa com Seres Humanos da Universidade Federal da Fronteira Sul (UFFS), obtendo o parecer número 2.222.266.

\section{RESULTADOS}

Foi identificado, no período de 2012 a 2016, uma totalidade de 3.084 notificações relativas aos acidentes de trabalho $\mathrm{e}$ doenças relacionadas ao trabalho, registradas no banco de dados do referido serviço envolvendo as mulheres trabalhadoras.

Tabela 1. Distribuição da variável faixa etária, segundo ano e sexo feminino, obtidas da coleta de dados do CEREST, no período de 2012-2016. Chapecó, SC, 2019.

\begin{tabular}{|c|c|c|c|c|c|c|c|c|c|c|c|c|}
\hline \multirow{2}{*}{ Faixa Etária } & \multicolumn{2}{|c|}{2012} & \multicolumn{2}{|c|}{2013} & \multicolumn{2}{|c|}{2014} & \multicolumn{2}{|c|}{2015} & \multicolumn{2}{|c|}{2016} & \multicolumn{2}{|c|}{ Total } \\
\hline & $\mathbf{N}$ & $\%$ & $\mathbf{N}$ & $\%$ & $\mathbf{N}$ & $\%$ & $\mathbf{N}$ & $\%$ & $\mathbf{N}$ & $\%$ & $\mathbf{N}$ & $\%$ \\
\hline 00 a 09 & 4 & 0,9 & 15 & 2,3 & 3 & 0,5 & 4 & 0,5 & 6 & 0,9 & 32 & 1,0 \\
\hline 10 a 19 & 20 & 4,7 & 34 & 5,2 & 36 & 6,0 & 27 & 3,7 & 18 & 2,7 & 135 & 4,4 \\
\hline 20 a 29 & 135 & 32,0 & 188 & 28,7 & 194 & 32,0 & 225 & 31,0 & 182 & 27,0 & 924 & 30,0 \\
\hline 30 a 39 & 122 & 29,0 & 216 & 33,0 & 177 & 29,2 & 208 & 29,0 & 219 & 32,4 & 942 & 30,5 \\
\hline 40 a 49 & 107 & 25,3 & 142 & 21,7 & 135 & 22,3 & 175 & 24,1 & 156 & 23,1 & 715 & 23,2 \\
\hline 50 a 59 & 30 & 7,1 & 52 & 8,0 & 59 & 9,7 & 75 & 10,3 & 76 & 11,2 & 292 & 9,5 \\
\hline 60 a 69 & 4 & 0,9 & 8 & 1,2 & 2 & 0,33 & 11 & 1,5 & 18 & 2,7 & 43 & 1,4 \\
\hline $\begin{array}{c}69 \text { anos e } \\
\text { mais }\end{array}$ & 0 & 0,0 & 0 & 0,0 & 0 & 0,0 & 1 & 0,1 & 0 & 0,0 & 1 & 0,03 \\
\hline Total & 422 & 100 & 655 & 100 & 606 & 100 & 726 & 100 & 675 & 100 & 3.084 & 100 \\
\hline
\end{tabular}


$\mathrm{O}$ maior número de registros no CEREST de acidentes e doenças de trabalho concentraram-se nas faixas etárias de 20 a 29 anos, de 30 a 39 anos e na faixa de 40 a 49 anos. É valido ressaltar que foram identificados casos de acidentes de trabalho nas faixas etárias de 00 a 09 e de 10 a 19 anos. Estes casos foram notificados como acidentes de trabalho com crianças e adolescentes, portanto não houve descrição da situação social em que se encontravam estes indivíduos, assim como a discussão sobre 0 trabalho infantil requer embasamento teórico em outros dados sociais, tal informação não foi neste artigo identificada.

Tabela 2. Distribuição da variável notificações do tipo de lesão, segundo ano e sexo feminino, obtidas da coleta de dados do CEREST, no período de 2012-2016. Chapecó, SC, 2019.

\begin{tabular}{ccccccccccc}
$\begin{array}{c}\text { Notificações do tipo } \\
\text { de lesão }\end{array}$ & \multicolumn{2}{c}{$\mathbf{2 0 1 2}$} & \multicolumn{2}{c}{$\mathbf{2 0 1 3}$} & \multicolumn{2}{c}{$\mathbf{2 0 1 4}$} & \multicolumn{2}{c}{$\mathbf{2 0 1 5}$} & \multicolumn{2}{c}{$\mathbf{2 0 1 6}$} \\
\cline { 2 - 12 } Trajeto & $\mathbf{N}$ & $\mathbf{\%}$ & $\mathbf{N}$ & $\mathbf{\%}$ & $\mathbf{N}$ & $\mathbf{\%}$ & $\mathbf{N}$ & $\mathbf{\%}$ & $\mathbf{N}$ & $\mathbf{\%}$ \\
\hline Lesão Cortante & 28 & 10,6 & 83 & 12,6 & 81 & 13,3 & 101 & 13,9 & 82 & 12,1 \\
\hline Lesão Perfurante & 23 & 5,6 & 96 & 14,6 & 142 & 23,4 & 138 & 19,0 & 115 & 17,0 \\
\hline Fraturas & 22 & 5,2 & 17 & 2,6 & 20 & 3,3 & 22 & 3,0 & 17 & 2,5 \\
\hline Queimadura & 11 & 2,6 & 16 & 2,4 & 28 & 4,6 & 28 & 3,8 & 22 & 3,2 \\
\hline Amputação & 3 & 0,7 & 9 & 1,3 & 2 & 0,3 & 3 & 0,4 & 5 & 0,7 \\
\hline Traumatismo & 50 & 11,8 & 163 & 24,8 & 174 & 28,7 & 232 & 31,9 & 216 & 32,0 \\
\hline Intoxicação & 19 & 4,5 & 62 & 9,4 & 56 & 9,2 & 50 & 6,9 & 14 & 2,0 \\
\hline Choque elétrico & 0 & 0,0 & 0 & 0,0 & 1 & 0,1 & 2 & 0,2 & 2 & 0,3 \\
\hline Penetração corpo & 0 & 0,0 & 0 & 0,0 & 6 & 1,0 & 6 & 0,8 & 5 & 0,7 \\
estranho & 0 & 0,0 & 0 & 0,0 & 0 & 0,0 & 5 & 0,7 & 1 & 0,14 \\
\hline Picada & 221 & 52,3 & 165 & 25,2 & 55 & 9,0 & 66 & 9,0 & 49 & 7,2 \\
\hline Não informado & 422 & 100 & 655 & 100 & 606 & 100 & 726 & 100 & 675 & 100
\end{tabular}

Conforme a tabela 2, ainda neste mesmo período de 2012 a 2016, observa-se nas notificações por tipo de lesão que a "lesão cortante" e o "traumatismo" tiveram relevância das demais em praticamente todos os anos. 
Tabela 3. Distribuição da variável notificações de acidente ou doença ocupacional, segundo ano e sexo feminino, obtidas da coleta de dados do CEREST, no período de 2012-2016. Chapecó, SC, 2019.

\begin{tabular}{|c|c|c|c|c|c|c|c|c|c|c|c|c|c|c|c|}
\hline \multirow{3}{*}{$\begin{array}{c}\text { Acidente ou } \\
\text { doença } \\
\text { ocupacional } \\
\text { Acidente com } \\
\text { material } \\
\text { biológico }\end{array}$} & \multicolumn{3}{|c|}{2012} & \multicolumn{3}{|c|}{2013} & \multicolumn{3}{|c|}{2014} & \multicolumn{3}{|c|}{2015} & \multicolumn{3}{|c|}{2016} \\
\hline & $\mathbf{N}$ & $\%$ & $\mathbf{T}$ & $\mathbf{N}$ & $\%$ & $\mathbf{T}$ & $\mathbf{N}$ & $\%$ & $\mathbf{T}$ & $\mathbf{N}$ & $\%$ & $\mathbf{T}$ & $\mathbf{N}$ & $\%$ & $\mathbf{T}$ \\
\hline & 42 & 82,3 & 51 & 54 & 81,8 & 66 & 48 & 78,6 & 61 & 79 & 84,0 & 94 & 130 & 76,0 & 171 \\
\hline LER/DORT & 99 & 69,2 & 143 & 91 & 68,4 & 133 & 46 & 57,5 & 80 & 61 & 70,0 & 88 & 48 & 69,5 & 69 \\
\hline $\begin{array}{l}\text { Intoxicações } \\
\text { exógenas }\end{array}$ & 5 & 83,3 & 6 & 15 & 71,4 & 21 & 18 & 64,2 & 28 & 27 & 57,4 & 47 & 13 & 52,0 & 25 \\
\hline $\begin{array}{l}\text { Acidente de } \\
\text { trabalho não } \\
\text { especificado }\end{array}$ & 265 & 26,8 & 987 & 446 & 25,0 & 1.778 & 438 & 21,0 & 2.091 & 0 & 0,0 & 0 & 0 & 0,0 & 0 \\
\hline Acidente fatal & 1 & 9,0 & 11 & 0 & 0,0 & 10 & 4 & 22,2 & 18 & 4 & 18,1 & 22 & 2 & 13,3 & 15 \\
\hline $\begin{array}{l}\text { Acidente com } \\
\text { mutilação }\end{array}$ & 3 & 17,6 & 17 & 1 & 4,5 & 22 & 3 & 12,0 & 25 & 3 & 13,0 & 23 & 4 & 15,3 & 26 \\
\hline $\begin{array}{l}\text { Acidente de } \\
\text { trabalho com } \\
\text { criança e } \\
\text { adolescente }\end{array}$ & 0 & 0,0 & 0 & 2 & 28,5 & 7 & 1 & 5,0 & 20 & 3 & 15,7 & 19 & 0 & 0,0 & 17 \\
\hline Acidente leve & 0 & 0,0 & 1 & 0 & 0,0 & 0 & 9 & 18,3 & 49 & 468 & 21,2 & 2.200 & 421 & 23,9 & 1.760 \\
\hline Acidente grave & 0 & 0,0 & 0 & 39 & 17,0 & 229 & 31 & 12,8 & 241 & 50 & 16,0 & 312 & 34 & 11,0 & 308 \\
\hline $\begin{array}{l}\text { Transtornos } \\
\text { mentais } \\
\text { relacionados ao } \\
\text { trabalho }\end{array}$ & 5 & 100 & 5 & 6 & 100 & 6 & 1 & 100 & 1 & 4 & 100 & 4 & 0 & 0,0 & 0 \\
\hline $\begin{array}{l}\text { Agravo bucal } \\
\text { relacionado ao } \\
\text { trabalho }\end{array}$ & 0 & 0,0 & 3 & 0 & 0,0 & 6 & 1 & 8,3 & 12 & 0 & 0,0 & 0 & 2 & 50,0 & 4 \\
\hline $\begin{array}{c}\text { Dermatose } \\
\text { ocupacional }\end{array}$ & 2 & 100 & 2 & 0 & 0,0 & 1 & 2 & 66,6 & 3 & 0 & 0,0 & 1 & 0 & 0,0 & 1 \\
\hline $\begin{array}{c}\text { Câncer } \\
\text { relacionado ao } \\
\text { trabalho }\end{array}$ & 0 & 0,0 & 0 & 0 & 0,0 & 0 & 0 & 0,0 & 0 & 0 & 0,0 & 0 & 1 & 100 & 1 \\
\hline Outros & 0 & 0,0 & 2 & 1 & 50,0 & 2 & 4 & 66,6 & 6 & 27 & 51,9 & 52 & 20 & 55,5 & 36 \\
\hline Total & & & 1.228 & & & 2.282 & & & 2.635 & & & 2.871 & & & 2.433 \\
\hline
\end{tabular}

Analisando-se o sexo feminino, que é por Esforço Repetitivo/Distúrbios o foco do estudo, percebe-se que as Osteomusculares Relacionados com o ocorrências que mais se destacaram com as trabalhadoras, de forma geral, foram os Trabalho (LER/DORT), as intoxicações exógenas e os acidentes de trabalho não acidentes com material biológico, as Lesões especificados. 
Tabela 4 - Distribuição da variável notificações pelo Código Brasileiro de Ocupações (CBO) no sexo feminino, obtidas da coleta de dados do CEREST, no período de 2012-2016. Chapecó, SC, 2019.

\begin{tabular}{|c|c|c|}
\hline CBO & $\mathbf{N}$ & $\%$ \\
\hline Alimentador de linha de produção & 439 & 14,2 \\
\hline Faxineiro & 363 & 11,7 \\
\hline Técnico de enfermagem & 294 & 9,5 \\
\hline Agente comunitário de saúde & 133 & 4,3 \\
\hline Cozinheiro geral & 121 & 3,9 \\
\hline Auxiliar de enfermagem & 115 & 3,7 \\
\hline Agente de saúde pública & 73 & 2,3 \\
\hline Enfermeiro & 65 & 2,1 \\
\hline Vendedor de comércio & 54 & 1,7 \\
\hline $\begin{array}{c}\text { Professor de nível superior do ensino } \\
\text { fundamental } * * *\end{array}$ & 48 & 1,5 \\
\hline Cozinheiro de hospital & 40 & 1,3 \\
\hline Auxiliar de escritório & 38 & 1,2 \\
\hline Servente de obras & 30 & 0,9 \\
\hline Pedreiro & 28 & 0,9 \\
\hline $\begin{array}{c}\text { Professor de nível superior na educação } \\
\text { infantil*** }\end{array}$ & 26 & 0,8 \\
\hline Assistente administrativo & 22 & 0,7 \\
\hline Auxiliar em serviços de alimentação & 21 & 0,6 \\
\hline $\begin{array}{l}\text { Professor da educação de jovens e adultos } \\
\text { do ensino fundamental }\end{array}$ & 19 & 0,6 \\
\hline $\begin{array}{c}\text { Professor de nível superior na educação } \\
\text { infantil* }\end{array}$ & 17 & 0,5 \\
\hline Médico clínico & 17 & 0,5 \\
\hline Cirurgião dentista & 13 & 0,4 \\
\hline Demais ocupações & 1.108 & 36 \\
\hline Total & 3.084 & 100 \\
\hline
\end{tabular}

Analisando as ocupações das mulheres trabalhadoras, a pesquisa apontou que 18,4\% delas pertencem à área da saúde, incluindo técnico de enfermagem, além do agente comunitário de saúde (ACS), auxiliar de enfermagem, agente de saúde pública, enfermeiro, médico clínico e cirurgião dentista.

\section{DISCUSSÃO}

De acordo com a tabela 1 a faixa etária de mulheres adultas trabalhadoras, no período de 2012 a 2016, demonstrou que o maior número de registros do CEREST quanto às notificações de acidentes e doenças de trabalho concentraram-se nas faixas etárias 20 a 29 anos, de 30 a 39 anos e na faixa de 40 a 49 , principalmente ao se analisar o percentual da faixa de 20 a 39 anos, destacando a fase produtiva $\mathrm{e}$ reprodutiva da mulher.

A população feminina vem passando por várias transformações na vida familiar, seus papeis e posições na sociedade. Apesar 
de todos os avanços prevalece a concepção da sociedade patriarcal que valoriza a mulher em razão de sua capacidade reprodutiva. A desigualdade social entre os gêneros tem reflexos no quadro de saúde de ambos os sexos e, em especial sobre a mulher. ${ }^{9}$

Conforme descrito na segunda tabela, apesar dos acidentes de trabalho representarem importante problema social, econômico e de saúde pública, esses eventos vêm sendo subnotificados. ${ }^{10}$ Ainda neste mesmo período de 2012 a 2016, observa-se nas notificações por tipo de lesão que a "lesão cortante" e o "traumatismo" tiveram destaque em praticamente todos os anos.

A ocorrência de acidentes do trabalho gera consequências traumáticas, podendo ocasionar mutilações, invalidez permanente, entre outros danos, que não se limitam ao corpo físico do trabalhador, afetando também sua integridade psicológica. $^{11}$

Com relação aos acidentes de trabalho, pode-se perceber que embora a promoção da segurança e da saúde dos trabalhadores tenha avançado nas últimas décadas, acidentes e doenças relacionados ao trabalho permanecem ainda elevados, penalizando fortemente os homens, as mulheres e suas famílias, mas pode-se perceber que o percentual de acidentes de trabalho envolvendo homens tem diminuído, em contrapartida os acidentes com mulheres teve um acréscimo, o que se explica através do ingresso das mulheres no mercado de trabalho. Com isso, à medida que as mulheres vêm ampliando sua participação no mercado de trabalho, surge também o acúmulo de funções e a dupla jornada de atividades, acumulando os afazeres domésticos e profissionais. Além disso, os perigos e os riscos que as mulheres enfrentam também estão relacionados com as condições de trabalho dos setores econômicos em que operam. ${ }^{12}$

Analisando-se o sexo feminino, que é o foco do estudo, percebe-se que as ocorrências que mais se destacaram com as trabalhadoras, foram os acidentes com material biológico, as LER/DORT, as intoxicações exógenas e os acidentes de trabalho não especificados.

As LER/DORT tem provocado sequelas irreversíveis aos trabalhadores que podem implicar em invalidez permanente. A dor e a fragilidade nos membros ou na coluna podem tornar-se crônicas e impossibilitar até mesmo a realização das tarefas mais simples do cotidiano, ocasionando o afastamento do trabalho devido aos limites e incapacidades gerados pela adversidade. ${ }^{14}$

As informações epidemiológicas são escassas sobre a mortalidade ou morbidade 
de intoxicações ocupacionais por agrotóxicos, geralmente há dificuldade de se identificar dados como tipo do agrotóxico utilizado, tipos de trabalho relacionado a intoxicação, e como o produto foi inoculado no organismo humano. ${ }^{15}$

$\mathrm{Na}$ categoria "Outros" agregaram-se as pneumoconioses, os acidentes por animais peçonhentos, o atendimento antirrábico humano, Leptospirose relacionada ao trabalho, a Brucelose relacionada ao trabalho, a Hantavirose relacionada ao trabalho, a Dengue relacionada ao trabalho e a violência relacionada ao trabalho.

A agroindústria apresenta-se como atividade mais representativa da economia do oeste catarinense, em especial o município de Chapecó, com o aumento dos frigoríficos. Portanto, ampliou-se a possibilidade de contratação de trabalhadores e esse aumento de empregos formais reflete mudanças nas estatísticas de notificação dos acidentes de trabalho.

No trabalho formal, no âmbito do chamado trabalho produtivo das empresas ligadas à produção de bens materiais, e nas empresas prestadoras de serviço, o trabalho de limpeza de imóveis (faxina) é o mais desqualificado, com baixos salários e pouca visibilidade social, apesar de na literatura cientifica estar descrito haver espaços de trabalho gratificantes para os profissionais que atuam neste ramo. ${ }^{16}$

Analisando as ocupações das mulheres trabalhadoras, foi identificado que 18,4\% delas pertencem à área da saúde, incluindo técnico de enfermagem, agente comunitário de saúde, auxiliar de enfermagem, agente de saúde pública, enfermeiro, médico clínico e cirurgião dentista.

Trabalhadores da área de enfermagem desenvolvem suas atividades em diversos locais, dentre eles os hospitais, local em que realizam atividades de forma contínua, que exigem atenção constante, esforço físico, movimentos repetitivos e levantamento de peso, o que os predispõem ao risco de adoecimento. $\mathrm{O}$ trabalho desses profissionais torna-se estressante em função da forte carga psicoemocional, das exigências físicas, do déficit de trabalhadores, dos turnos prolongados e das condições inadequadas de trabalho. ${ }^{14}$

\section{CONCLUSÃO}

É notório, a partir desta investigação, que as mulheres também são vulneráveis em relação aos acidentes/doenças relacionadas ao trabalho. $\mathrm{O}$ perfil em destaque são as mulheres em idade produtiva para o mercado de trabalho, que principalmente sofrem lesões cortantes ou traumatismo devido a exposição a 
materiais biológicos, LER/DORT e intoxicações exógenas, sobretudo por atuarem com atividades como alimentação de linha de produção, faxineira e técnico de enfermagem.

Propõe-se a necessidade de políticas de trabalho/saúde que visem a proteção e promoção da saúde da mulher trabalhadora, tendo em vista que esta, no cenário social atual, possui duas jornadas de trabalho, uma formal e outra informal, sendo esta última o cuidado do lar. Logo, implicações a sua saúde incide em mais de uma dimensão social.

O estudo limitou-se a dados disponibilizados pelo CEREST com período de atraso na notificação dos dados. Além disso, pode ocorrer subnotificação das informações. Sendo assim, consideramos que o perfil aqui estabelecido possui limitações no que tange generalizações.

\section{REFERÊNCIAS}

1. Girelli S, Magro MLPD. Saúde do trabalhador e economia solidária: estudo de uma cooperativa de construção civil. Fractal Rev Psicol. [Internet]. 2017 [citado em 13 fev 2019]; 29(1):9-16. doi: http://dx.doi.org/10.22409/19840292/v29i1/1184

2. Araújo TMP, Araújo TF, Carmo N. Vigilância em Saúde Mental e Trabalho no Brasil: características, dificuldades e desafios. Ciênc Saúde Colet. [Internet]. 2017 [citado em 13 fev 2019]; 22(10):
3235-46. doi: https://doi.org/10.1590/1413812320172210.17552017

3. Edilmar M. Implantação de equipe de saúde do trabalhador nas empresas: um olhar sobre doenças ocupacionais com manifestação bucal. Braz Ap Sci Rev. [Internet]. 2018 [citado em 13 fev 2019]; 25(2):568-82. Disponível em: http://www.brazilianjournals.com/index.ph p/BASR/article/view/422/362

4. Silva A, Ferraz L, Rodrigues-Junior SA. Ações em saúde do trabalhador desenvolvidas na atenção primária no município de Chapecó, Santa Catarina. Rev Bras Saúde Ocup. [Internet]. 2016 [citado em 13 fev 2019]; 41:e16. doi: http://dx.doi.org/10.1590/23176369000122415

5. Oliveira LA, Oliveira EL. A mulher no mercado de trabalho: algumas reflexões. Rev REFAF Multidisciplinar [Internet]. 2019 [citado em 13 fev 2019]; 8(1):1-15. Disponível em:

http://refaf.com.br/index.php/refaf/article/v iew/287/pdf

6. Portal Brasil (Brasil). Mulheres são mais vulneráveis a doenças no ambiente de trabalho [Internet]. Brasília, DF: Ministério da Previdência; 2020 [citado em 07 jun 2020]. Disponível em: https://www.gov.br/previdencia/ptbr/assuntos/noticias/previdencia/conselhode-previdencia/cnps-mulheres-sao-maisvulneraveis-a-doencas-causadas-pelotrabalho

7. Hochman B, Nahas FX, Oliveira Filho RS, Ferreira LM. Desenhos de pesquisa. Acta Cir Bras. (Online) [Internet]. 2005 [citado em 20 fev 2019]; 20 (supl 2):2-9. Disponível em: http://www.scielo.br/pdf/acb/v20s2/v20s2a 02.pdf

8. Instituto Brasileiro de Geografia e Estatística (Brasil). Cidades [Internet]. Rio de Janeiro: IBGE; 2016 [citado em $20 \mathrm{fev}$ 2019]. Disponível em: https://cidades.ibge.gov.br/brasil/sc/chapec o/panorama 9. Hirata H. Globalização, trabalho e gênero. Rev Polít Públicas [Internet]. 2005 
[citado em 23 fev 2019]; 9(1):111-28.

Disponível em:

http://www.periodicoseletronicos.ufma.br/i ndex.php/rppublica/article/view/3770/1848 10. Mendes JMR, Wunsch DS, Machado FKS, Martins J, Giongo CR. Saúde do trabalhador: desafios na efetivação do direito à saúde. Argum. [Internet]. 2015 [citado em 23 fev 2019]; 7(2):194-207. Disponível em:

https://periodicos.ufes.br/argumentum/artic le/view/10349/8253

11. Bastos-Ramos TP, Santana VS, Ferrite S. Estratégia Saúde da Família e notificações de acidentes de trabalho, Brasil, 2007-2011. Epidemiol Serv Saúde [Internet]. 2015 [citado em 23 fev 2019]; 24(4):641-50. Disponível em:

http://www.scielo.br/scielo.php?script=sci _arttext\&pid=S2237-96222015000400641

12. Souza TV. A influência do sexo e do gênero nas questões envolvendo segurança e saúde do trabalhador. Revista Espaço Acadêmico [Internet]. 2016 [citado em 03 abr 2019]; (177):73-83. Disponível em: http://periodicos.uem.br/ojs/index.php/Esp acoAcademico/article/view/28794/16162 13. Brust RS, Oliveira LPM, Silva ACSS, Regazzi ICR, Aguiar GS, Knupp VMAO. Perfil epidemiológico de trabalhadores rurais do estado do Rio de Janeiro. Rev Bras Enferm. [Internet]. 2019 [citado em 03 abr 2019]; 72 ( Suppl 1 ):129-35. doi: http://dx.doi.org/10.1590/0034-7167-20170555

14. Viegas LRT, Almeida MMC. Perfil epidemiológico dos casos de LER/DORT entre trabalhadores da indústria no Brasil no período de 2007 a 2013. Rev Bras Saúde Ocup. [Internet]. 2016 [citado em 03 abr 2019]; 41:e22. doi: http://dx.doi.org/10.1590/23176369000130615 15. Padilha NS. Agrotóxicos e a saúde do trabalhador: a responsabilidade por contaminação do meio ambiente do trabalho. Rev Jurídica Luso-Brasileira [Internet]. 2018 [citado em 03 abr 2019]; 4(6): 977-1006. Disponível em: http://www.cidp.pt/revistas/rjlb/2018/6/201 8_06_0977_1006.pdf

16. Alvarez D, Azevedo ERF. O trabalho feminino na função de limpeza de prestadoras de serviço em uma instituição de ensino superior. Rev Vianna Sapiens [Internet]. 2016 [citado em 03 abr 2019]; 7(1):185-212. Disponível em: http://viannasapiens.com.br/revista/article/ view/188

RECEBIDO: 10/05/2019

APROVADO: $20 / 03 / 2020$

PUBLICADO: 07/2020 